\title{
NOUVELle
}

\section{TP53 et RB collaborent dans le développement des rétinoblastomes humains}

Olivier Ayrault, Frédérique Zindy, Martine F. Roussel

\author{
Department of Genetics and Tumor Cell Biology, \\ St. Jude Children's Research Hospital, Memphis, \\ mail stop 350, 332 North Lauderdale, \\ Memphis, 38105 Tennessee, TN, États-Unis. \\ martine.roussel@stjude.org
}

> Les voies régulatrices $\mathrm{pl} 6^{\text {INK4A } / C Y C L I N E ~ D / ~}$ CDK4-6/pRB (RB, rétinoblastome) et $\mathrm{pl} 4^{\mathrm{ARF} /}$ MDM2-MDMX/TP53 (TP53) sont aujourd'hui bien connues pour leur rôle anti-tumoral. Elles induisent l'arrêt du cycle cellulaire, la sénescence ou l'apoptose des cellules cancéreuses [1]. Localisés sur le même locus génomique en $9 \mathrm{p} 21$, les deux gènes humains suppresseurs de tumeurs INK4A et ARF ont la caractéristique, quasi-unique chez les eucaryotes, de donner naissance, à partir de promoteurs distincts et de leurs trois exons (exons 1 alternatifs $\alpha$ [pour INK4A] et $\beta$ [pour ARF], exons 2 et 3 identiques), à deux protéines sans aucune parenté qui se comportent comme des régulateurs négatifs de la prolifération. $\mathrm{Pl} 6^{\mathrm{INK} 4 \mathrm{~A}}$ régule négativement la voie RB tandis que pl4 ${ }^{A R F}$ permet l'activation de la voie TP53 (Figure IA). Chez I'homme, dans la plupart des cancers, les voies régulatrices $\mathrm{RB}$ et TP53 sont altérées. Ces altérations représentent, par conséquent, une étape importante et peut-être requise, dans le développement tumoral. La mutation du gène $R B I$ conduit à la formation du rétinoblastome, cancer de la rétine de l'enfant [2]. Classiquement, il existe deux types de rétinoblastome: l'un à transmission héréditaire dans lequel l'un des allèles muté est transmis à l'enfant. L'inactivation de l'autre allèle peut conduire à un rétinoblastome multifocal dans lequel les deux yeux sont touchés. L'autre cas, sporadique, dans lequel les deux allèles sont mutés dans le même œil, conduit à un rétinoblastome unilatéral. $\mathrm{RB} 1$ fait partie de la famille des protéines pockets qui comprend également p107 et p130. Contrairement à RB1, p107 et p130 ne sont pas mutés dans le rétinoblastome humain. Pour essayer de mieux comprendre ce type de cancer, plusieurs modèles murins ont été développés. Ces modèles reproduisent les caractéristiques de la tumeur humaine, mais nécessitent l'inactivation de deux membres de la famille $R b: R b$ et $p 107$. La fréquence et la pénétrance des tumeurs sont fortement augmentées lorsque p53 est également inactivé. Cela suggère que la perte de $p 53$ peut jouer un rôle important dans le développement du rétinoblastome. Cependant, TP53 n'a jamais été trouvé muté dans les retinoblastomes humains. On a donc supposé que la voie TP53 n'était pas requise pour l'inactivation tumorale et que les cellules de la rétine contenant une mutation de $R B I$ étaient résistantes à l'activité apoptotique de TP53.

Le taux de TP53 dans la cellule est notamment contrôlé par pl4 ${ }^{\mathrm{ARF}}$ et MDM2. P14 ${ }^{\mathrm{ARF}}$, exprimée lorsque les signaux prolifératifs sont trop élevés, se lie à MDM2 et MDMX et empêche ces derniers de dégrader TP53 (Figure 1A). MDM2 se lie à TP53 et, grâce à son activité d'ubiquitine-ligase ( $\varepsilon 3)$, permet sa dégradation via le protéasome. MDMX (aussi connu sous le nom de MDM4), un autre membre de la famille de MDM2, possède les mêmes caractéristiques que MDM2. Comme MDM2, MDMX est également fréquemment surexprimée dans plusieurs types de tumeurs. Les premières données montrant que la voie p14 ARF/MDM2-MDMX/TP53 joue un rôle dans le développement du rétinoblastome proviennent d'une étude récente réalisée par l'équipe du Docteur Michael Dyer [3, 4] (St. Jude Children's Research Hospital, Memphis, États-Unis). L'analyse de tumeurs humaines (rétinoblastomes) a montré un taux de pl4 ${ }^{\text {ARF }} 71$ à 500 fois plus important que celui qui est observé dans des rétines fœtales normales. Sachant que la protéine TP53 est fonctionnelle dans le rétinoblastome, il était logique de penser qu'un taux aussi important de $\mathrm{pl} 4^{\mathrm{ARF}}$ conduirait à une stabilisation de TP53 permettant ainsi un arrêt du cycle cellulaire ou l'apoptose des cellules rétiniennes tumorales. Cependant, la protéine TP53 n'est pas du tout stabilisée dans ces tumeurs. Cela suggérait donc fortement qu'il devait y avoir un autre gène dans la voie TP53 dont l'expression devait être altérée. Des analyses par arrayCGH (comparative gene hybridization) sur des tumeurs humaines ont montré que MDMX est amplifié (3/7). Cette amplification est corrélée à une augmentation de l'ARN et de la protéine MDMX. Par analyse FISH (fluorescent in situ hybridization), 32 tumeurs sur 49 (65\%) montrent des copies supplémentaires de MDMX et 5 tumeurs sur 49 (10\%) de MDM2. Cela suggérait que l'hyper-expression de MDMX et de MDM2 induit une dégradation de TP53 dans les cellules tumorales, même en présence d'un taux élevé de pl4 ${ }^{\mathrm{ARF}}$, qui serait par conséquent insuffisant pour la séquestration complète de MDMX et MDM2. En effet, la surexpression de MDMX, dans des cellules de rétine de souris nouveau-nées invalidées pour $R b$ et $p 107$, induit la prolifération et la survie des cellules rétiniennes mais également l'apparition de rétinoblastomes, dont l'agressivité est comparable à celle des tumeurs provenant des souris invalidées pour $p 107, R b$ et $p 53$. De plus, la surexpression de MDMX et l'inactivation de $R B I$ dans des cellules de rétines normales humaines induisent l'organisation des cellules en rosettes comparables à celles qui sont observées dans les rétinoblastomes humains.

L'équipe du Dr M. Dyer a également montré qu'en aval de MDMX, la réponse 
aux dommages à I'ADN faisant intervenir TP53 est bien fonctionnelle dans les rétinoblastomes. En effet, l'irradiation des cellules tumorales ou leur traitement par Topotécan [5], agent chimio-thérapeutique inhibiteur de la topoisomérase I, permet l'induction de TP53 et, par conséquent, l'apoptose. Cette apoptose est accrue par l'inactivation de MDMX. Le traitement associant la Nutline-3, petite molécule inhibitrice de la liaison entre TP53 et MDMX, et Topotécan tue les cellules de rétinoblastomes. De plus, dans un modèle de transplantation de cellules de rétinoblastomes humains chez la souris, l'injection simultanée par voie sous-conjonctivale de ces deux drogues permet une réduction des rétinoblastomes, sans effet secondaire contrairement à I'utilisation de ces deux drogues par voie systémique (Figure lB). Cette découverte de l'équipe de $M$. Dyer permet d'envisager un traitement des patients par injection sous-conjonctivale de Topotécan et Nutline-3. Ce type d'administration pourrait permettre

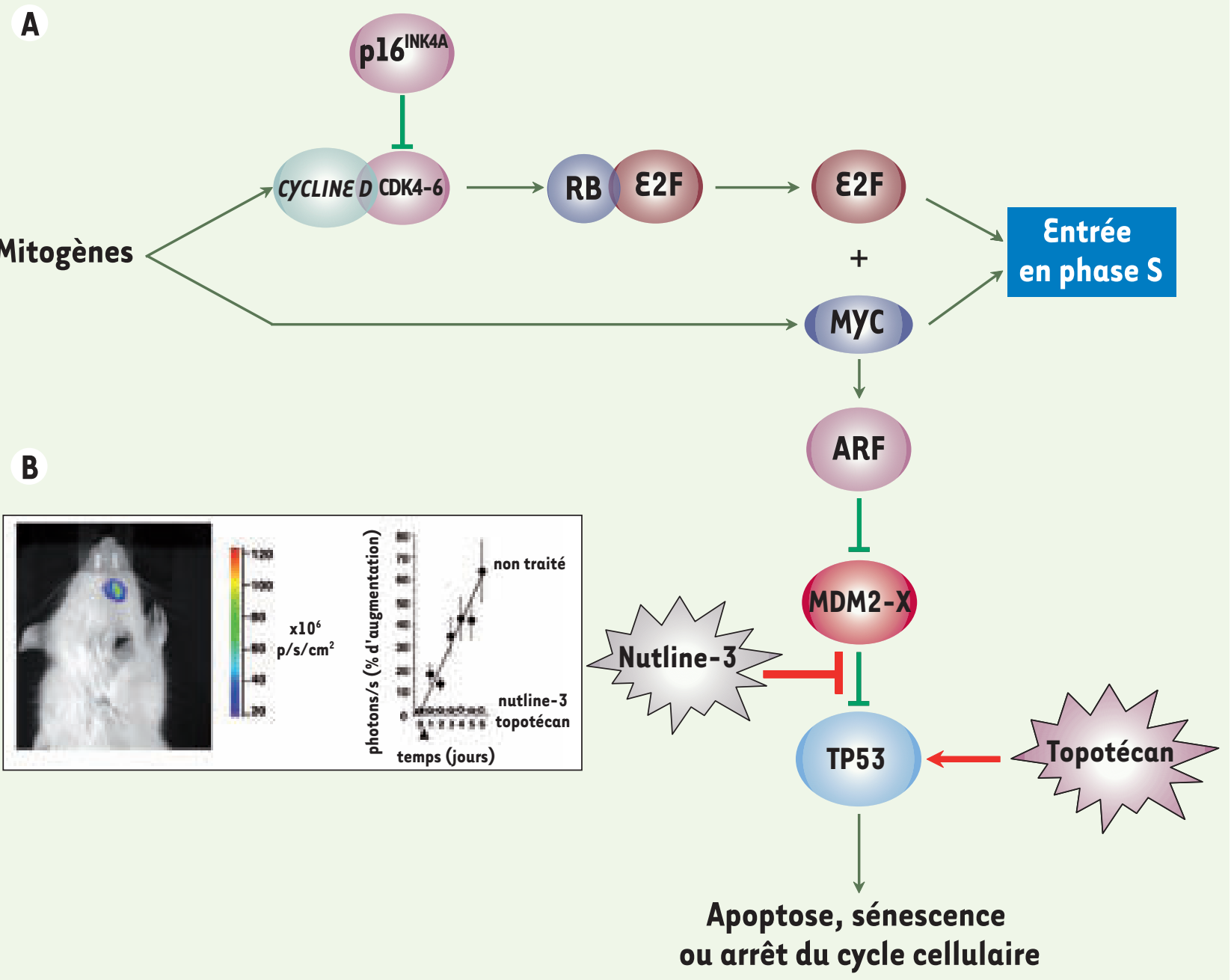

Figure 1. A. Représentation schématique des voies régulatrices $p 16^{I N K 4 A} / C Y C L I N \varepsilon D / C D K 4-6 / p R B$ et $p 14^{A R F / M D M 2-M D M X / T P 53 . ~ S o u s ~ I ' a c t i o n ~ d e ~}$ mitogènes, la CYCLINE D est synthétisée et se lie aux kinases CDK4-6. La phosphorylation de RB par CDK4-6 permet la libération des facteurs de transcription $\varepsilon 2 F$ du complexe RB/E2F. Les $\varepsilon 2 F$ libres activent la transcription de gènes nécessaires à l'entrée en phase $S$. દ2F et MYC induisent p14ARF. $\mathrm{P} 14^{\mathrm{ARF}}$ se lie à MDM2 et MDMX et empêche ces derniers de dégrader TP53. L'accumulation de TP53 induit l'apoptose, la sénescence ou l'arrêt du cycle cellulaire. Nutline-3 inhibe la liaison entre TP53 et MDM2-MDMX. Topotécan permet l'induction de TP53 en provoquant des dommages de l'ADN. B. L'injection subconjunctivale simultanée de Nutline-3 et Topotécan permet une réduction des rétinoblastomes humains. Des cellules de rétinoblastomes humains exprimant constitutivement la luciférase ont été injectées dans les yeux de souris immunodéficientes. Les souris non traitées développent un rétinoblastome, visualisé par la luciférase (photo et cercles noirs sur le graphique de droite) alors que les souris traitées par la Nutline-3 ou le Topotécan développent un rétinoblastome de taille inférieure. L'administration simultanée des deux drogues (cercles ouverts sur le graphique de droite) permet une réduction encore plus importante des rétinoblastomes (photographie donnée par M. Dyer). 
d'obtenir de très bons résultats sur la régression du rétinoblastome, et surtout, d'éviter les profonds effets secondaires induits par les chimiothérapies. $\diamond$ TP53 and RB tumor suppressor pathways collaborate in retinoblastoma genesis

\section{REMERCIEMENTS}

Nous remercions Michael Dyer pour nous avoir donné la Figure 1B. Olivier Ayrault est financé par la Fondation pour la Recherche Médicale
(09/05-09/06) et par le Gephardt Endowed Fellowship-Signal Transduction (09/0609/08). Frédérique Zindy voudrait remercier très sincèrement le professeur Gérard Buttin pour son support moral tout au long de sa carrière scientifique. Martine F. Roussel est financée par the NIH CA-96832, Core Grant CA21765, the Children's Brain Tumor Foundation (CBTF) et the Pediatric Brain Tumor Foundation (PBTF), the American-Lebanese Syrian Associated Charities (ALSAC) and St. Jude Children's Research Hospital.

\section{NOUVELLE}

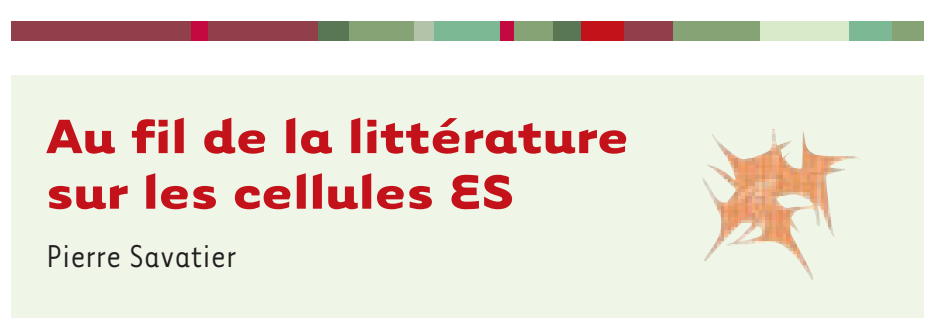

$>$ En 2005-2006, la recherche sur les cellules souches embryonnaires (દS) a été marquée par deux avancées technologiques majeures pouvant avoir un impact en thérapie cellulaire ( $d$ 'autres avancées fondamentales dans ce domaine des cellules ES ont été traitées par ailleurs récemment dans Médecine/Sciences) [1-3]. La première est la dérivation de lignées de cellules souches pluripotentes à partir de cellules somatiques chez la souris. L'équipe de Shinya Yamanaka (Nara, Japon) a obtenu la re-programmation de fibroblastes embryonnaires et adultes grâce à la surexpression de quatre facteurs de transcription, 0ct4, Sox2, Myc et KIf4 [4]. Ces facteurs sont connus pour leur rôle dans le maintien des cellules ES à l'état pluripotent chez la souris ( $0 \mathrm{ct} 4$, Sox2, Myc, KIf4) et chez l'homme (0ct4, Sox2). Les cellules pluripotentes ainsi obtenues sont appelées iPS (induced pluripotent stem) afin de les différencier des véritables cellules ES obtenues à partir d'embryons. Les cellules iPS présentent les caractéristiques cardinales de cellules $\varepsilon S$, en particulier un potentiel de prolifération illimité et la capacité de différenciation en cel- lules des trois feuillets embryonnaires, ectoderme, mésoderme et endoderme, et ce en l'absence d'anomalies chromosomiques. On ne peut exclure, ni que les cellules ainsi reprogrammées sont des cellules souches tissulaires résiduelles (et non des fibroblastes différenciés), ni que ce processus de re-programmation nécessite un ou plusieurs évènements secondaires de nature inconnue, car l'efficacité de cette re-programmation est faible. Néanmoins, ce travail fait la preuve du concept de re-programmation directe d'une cellule somatique en cellule souche pluripotente. La preuve de ce concept doit maintenant être faite chez l'homme. La capacité de cellules iPS humaines à s'autorenouveler sans altération génétique dans un milieu de culture dépourvu de composants d'origine animale, puis à se différencier in vitro en cellules d'intérêt thérapeutique, devra être étudiée et confirmée. L'efficacité de la re-programmation devra être augmentée, sans doute grâce à l'ajout d'autres gènes de pluripotence ou à l'utilisation d'inhibiteurs pharmacologiques de diverses voies de signalisation impliquées dans la différenciation. À

\section{RÉFÉRENCES}

1. Sherr CJ, McCormick F. The RB and $p 53$ pathways in cancer. Cancer Cell 2002 ; 2 : 103-12.

2. Classon $M$, Harlow $\varepsilon$. The retinoblastoma tumour suppressor in development and cancer. Nat Rev Cancer $2002 ; 2: 910-7$.

3. Dyer MA, Rodriguez-Galindo C, Wilson MW. Use of preclinical models to improve treatment of retinoblastoma. PLoS Med $2005 ; 2$ : e332.

4. Laurie NA, Donovan SL, Shih CS, et al. Inactivation of the $p 53$ pathway in retinoblastoma. Nature 2006 ; $444: 61-6$.

5. Laurie NA, Gray JK, Zhang J, et al. Topotecan combination chemotherapy in two new rodent models of retinoblastoma. Clin Cancer Res 2005 ; 11 : 7569-78.
Inserm U846,

Institut Cellules souches et Cerveau, 18, avenue Doyen Lépine, 69500 Bron, France. savatier@lyon.inserm.fr

terme, cette technologie est susceptible de permettre la production de cellules différenciées histocompatibles sans passer par l'étape du transfert nucléaire et la constitution d'embryons clonés. Par ailleurs, cette avancée technologique illustre la nécessité de poursuivre les recherches sur les mécanismes de contrôle de l'autorenouvellement et de la pluripotence des cellules ES afin, à terme, de maîtriser totalement le processus de re-programmation.

La deuxième avancée concerne l'identification de facteurs de croissance (et des voies de signalisation correspondantes) capables de maintenir les cellules ES humaines (HES) dans un état d'autorenouvellement. Plusieurs travaux réalisés par les équipes de Jamie Thomson (Université du Wisconsin, États-Unis) $[5,6]$, Ali Hemmati-Brivanlou (Université Rockefeller, États-Unis) [7] et Roger Pedersen (Cambridge, Royaume-Uni) [8, 9] ont démontré le rôle de l'Activine et de Nodal (facteurs de la famille du TGF- $\beta$, transforming growth factor $\beta$ ), ainsi que des facteurs FGF2 et Wnt, dans la stimulation de l'autorenouvellement et l'inhibition de la différenciation. En revanche, 